\title{
Analysis of the Relationship between the Number of Traffic Accidents and the Traffic Flow \& Section Location in Extra Long Tunnel
}

\author{
Zhaoyou Ma1,2, Shouen Fang ${ }^{1}$, Shuo Liu ${ }^{1 *}$, Bowen Cai ${ }^{1}$ \\ ${ }^{1}$ Key Laboratory of Road and Traffic Engineering of the Ministry of Education, Tongji University, Shanghai, China \\ ${ }^{2}$ Key Laboratory of Road Traffic Safety of the Ministry of Public Security, Traffic Management Research Institute of \\ Public Security Ministry, Wuxi, China \\ Email: uarain@126.com, fangsek@mail.tongji.edu.cn, *avensonliu@163.com, caibowen@tongji.edu.cn
}

How to cite this paper: Ma, Z.Y., Fang, S.E., Liu, S. and Cai, B.W. (2020) Analysis of the Relationship between the Number of Traffic Accidents and the Traffic Flow \& Section Location in Extra Long Tunnel. Engineering, 12, 71-81.

https://doi.org/10.4236/eng.2020.122007

Received: December 19, 2019

Accepted: January 31, 2020

Published: February 3, 2020

Copyright $\odot 2020$ by author(s) and Scientific Research Publishing Inc. This work is licensed under the Creative Commons Attribution International License (CC BY 4.0).

http://creativecommons.org/licenses/by/4.0/

\begin{abstract}
In order to study the influence of the traffic characteristics on traffic accidents in extra long tunnel, the main measurement indicators of traffic flow during the time of traffic accidents are matched with the accident information to form a data set of the number of traffic accidents and the hourly traffic flow of the accident. Vehicle ratio and the number of accidents are mainly used as the characteristic indicators of traffic flow. At the same time, the longitudinal distribution law of the average speed of traffic flow and the number of traffic accidents in the extra long tunnel is studied. Based on the superposition principle, the extra long tunnel is divided into 5 traffic safety zones. This paper analyzes the distribution of time, morphology, cause of accident, and other characteristics in different traffic safety zones, finding that the shape of traffic accidents in extra long tunnel is mainly rear-end collisions. Improper operation and illegal lane changes are the main causes of accidents.
\end{abstract}

\section{Keywords}

Traffic Safety, Extra Long Tunnel, Traffic Accident, Accident Analysis

\section{Introduction}

China has the largest number of tunnel projects, the most complicated conditions, and the fastest technological development in the world [1]. In China, by the end of 2018 , there were 17,738 road tunnels with a total of 17.2361 million meters long, wherein 1058 tunnels were extra long tunnels with a total of 4,706,600 meters [2]. With the increasing mileage of tunnels, tunnel traffic safety problems have become increasingly apparent, and road tunnels have become 
traffic blackspots with a high frequency of accidents. From 2011 to 2018, the fatality rate of tunnel accidents was as high as 0.48 persons/case, which is 1.6 times the mortality rate of all accidents in the same period. An in-depth analysis of the rules of tunnel traffic accidents, and excavation of the internal factors that affect the accidents, is of great significance to the operation and management of tunnels and the prevention of traffic accidents. The tunnel-related research results rarely involve the longitudinal distribution of traffic flow and traffic accidents in long tunnel. Zhou Jingjing [3] studied the correlation between the distribution of tunnel accident sections and the characteristics of the tunnel environment. Yeung et al. [4] studied the spatial distribution characteristics of the degree. Tanishita [5] studied the relationship between the speed change and the traffic accident rate. This paper focuses on analyzing the correlation between the number of traffic accidents and traffic flow characteristics and longitudinal position of the extra long tunnel.

\section{Tunnel Definition and Classification}

Tunnels are defined as "built underground (or through the mountain) for vehicular access or both non-motor vehicles and pedestrian traffic features of road structures", according to industry standards such as "Specification for design of highway tunnels" (JTG D70-2014) and "Code for design of urban underground road engineering" (CJJ 221-2015).

According to the nature of the use of tunnel, it can be classified into highway tunnel and urban tunnel. However, in light of the length of the tunnel, tunnels can be classified into four types: extra long tunnel, long tunnel, medium tunnel and short tunnel, as shown in Table 1.

In addition, due to the different topography, tunnels can be classified into mountain tunnel, underwater tunnel; in accordance with their cross-sectional shapes, tunnels can be classified into round tunnel, oval tunnel and horseshoe tunnel.

\section{Longitudinal Distribution of Traffic Flow and Number of Accidents in Extra Long Tunnel}

\subsection{Longitudinal Distribution of Traffic Flow Speed in Extra Long Tunnel}

In this study, a total length of 10 tunnels in a certain area were selected. Six kinds of feature points in $200 \mathrm{~m}$ (JW200) outside of tunnel entrance, entrance position (JK), $300 \mathrm{~m}$ (JN300) within the entrance, $300 \mathrm{~m}$ (CN300) before the exit, $100 \mathrm{~m}$

Table 1. Tunnel length classification.

\begin{tabular}{ccccc}
\hline Tunnel Classification & Extra Long Tunnel & Long Tunnel & Middle Tunnel & Short Tunnel \\
\hline Length L (m) & L $>3000$ & $3000 \geq \mathrm{L}>1000$ & $1000 \geq \mathrm{L}>500$ & $\mathrm{~L} \leq 500$
\end{tabular}

Note: The tunnel length L refers to the distance between the intersection of the two ends of the door-end wall surface and the road surface and the intersection of the center line of the route. 
(CN100) before the exit, and $100 \mathrm{~m}$ outside the exit (CW100) were respectively chosen and get installed with an automatic speed measuring device. The setting period is from 11 to 14 noon. To ensure free flow, the head time interval was selected to be greater than 8 seconds. Finally, the speed cumulative curve of each feature point was obtained. The running speed of the feature point was $85 \%$ of the vehicle speed.

It can be seen from Figure 1 that the speed of the test vehicle dropped sharply from JW200 to JK, reaching the lowest level at the exit of the tunnel. After entering the tunnel, the driving speed gradually increased and became relatively stable at around JN300. It then traveled at a steady speed to CN300, where the driving speed suddenly boomed and dropped sharply all the way to the minimum speed at the tunnel $\mathrm{CN} 100$, After $\mathrm{CN100}$, the test vehicle accelerated to get out of the tunnel.

It can be seen from Figure 2 that the test vehicle speed varied largely in the



Figure 1. Characteristic speed distribution of different feature points of extra long tunnel.

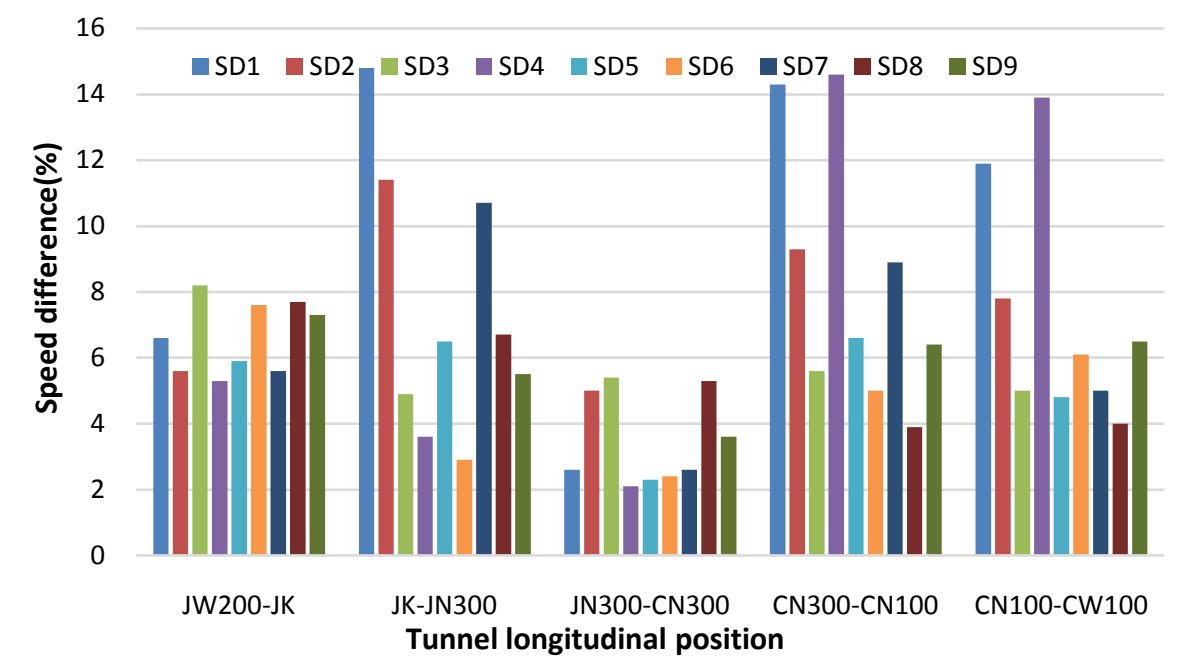

Figure 2. Variation of operating speed difference between adjacent feature points in extra long tunnel. 
sections JN200-JK, JK-JN300, CN300-CN100, and CN100-CW100, and the speed variation in JN300-CN300 was smaller. When approaching to the tunnel, as the distance to the tunnel entrance was lessening, the driver would reduce the vehicle operation speed; after entering the tunnel, after a short period of adaptation, the vehicle operation speed would increase a bit, but the speed inside the tunnel was still lower than that outside the tunnel.

In summary, after the vehicle entered the extra long tunnel, when the driver gradually adapted to the internal environment of the tunnel, the driving speed was obviously increased, and the vehicle traveled at a relatively constant speed for a distance until it entered the end of the tunnel. As the vehicle was about to leave the tunnel, the speed was reduced to some extent. After the vehicle left the tunnel, there was often an acceleration behavior [6].

\subsection{Longitudinal Distribution of Traffic Accidents in Extra Long Tunnel}

A total of 6 up and down road tunnels of a certain area were selected, with the tunnel entrance and tunnel exit as the origin with $0.1 \mathrm{~km}$ as the unit. The distribution of traffic accidents in the past 3 years was counted. Taking TCSD1 (double-hole 6 lanes, full length $8.9 \mathrm{~km}$ ) and TCSD2 (double-hole 4 lanes, total length $3.6 \mathrm{~km}$ ) as an example, the longitudinal distribution of traffic accidents in extra long tunnel was analyzed.

It can be seen from Figure 3 that the last sudden change of the longitudinal distribution of traffic accidents outside the entrance of the extra long tunnel occurred at $0.1 \mathrm{~km}$ from the entrance. Within this zone, the speed of the section was obviously changed, the lane change behavior was quite frequent, and the traffic signs were set unreasonably, which as a result, leading to the distraction of driver. The longitudinal distribution of traffic accidents within $1 \mathrm{~km}$ of the entrance tunnel of the extra long tunnel was similar. The first obvious sudden change point occurred at $0.3 \mathrm{~km}$ from the entrance, wherein the driver was adjusting the speed to adapt to the tunnel light environment. Therefore, the proportion of accidents due to the improper operation was significant, showing that the $0.1 \mathrm{~km}$ outside the entrance tunnel of the extra long tunnel and the $0.3 \mathrm{~km}$ inside the tunnel were affected largely by the drivers' interaction with the tunnel.

It can be seen from Figure 4 that the distribution of traffic accidents in the exit tunnel of the extra long tunnel fluctuated greatly, and the last sudden change point occurred at $0.1 \mathrm{~km}$ from the tunnel exit. The driver was affected by the tunnel's depressing environment for a long duration. The feeling of getting out of the tunnel rapidly was, therefore, becoming more urgent. Hence, with the influence of the tunnel exit, traffic accidents were easily caused due to paralysis and improper operation. The longitudinal distribution of traffic accidents outside the tunnel of the extra long tunnel was highly fluctuating from $0.1 \mathrm{~km}$ to 0.3 $\mathrm{km}$. The driver of this section gradually adapted to the environment outside the tunnel after passing through the tunnel exit. Compared to tunnel environment, driver was getting more relaxed and the driving speed was easily to get fast outside 


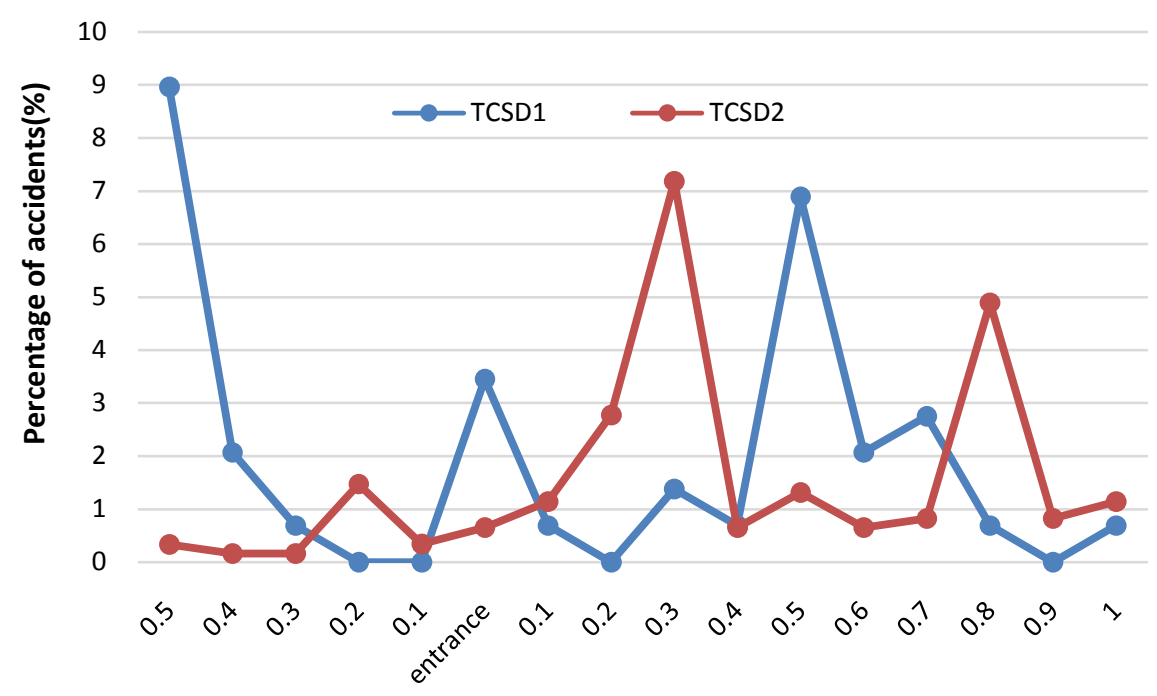

Accident point location(diatance to entrance, $\mathrm{km}$ )

Figure 3. Distribution of traffic accidents at the entrance of extra long tunnel.

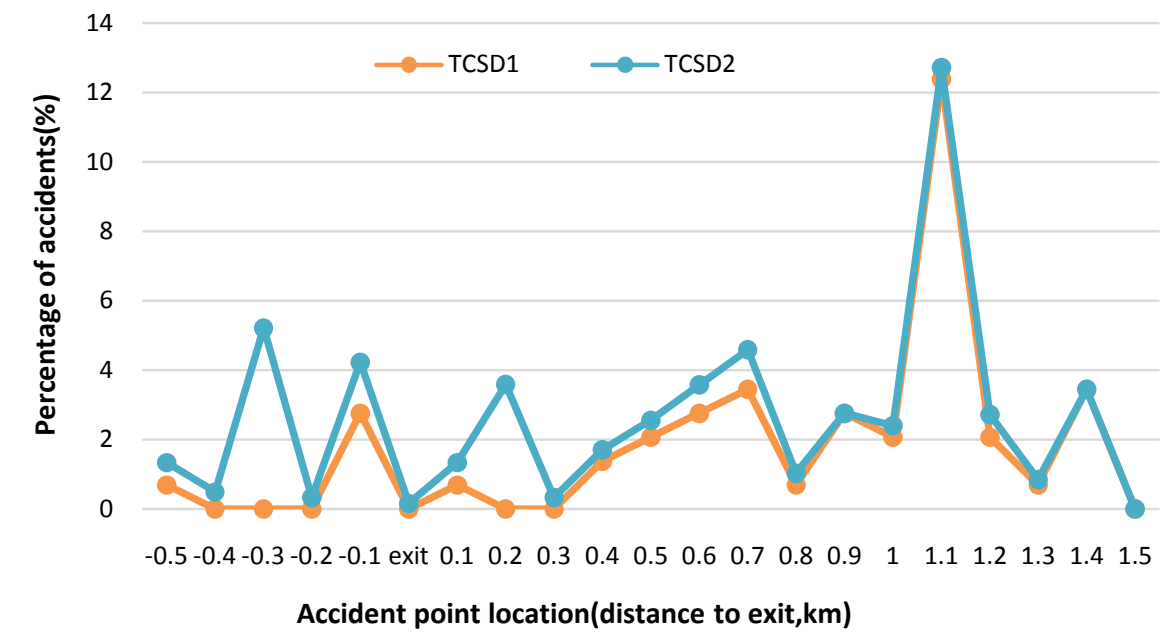

Figure 4. Distribution of traffic accidents at the exit of the extra long tunnel.

of tunnel, indicating that the traffic accidents within $0.1 \mathrm{~km}$ to $0.3 \mathrm{~km}$ outside of the extra long tunnel exit was affected by the driver interaction with tunnel significantly.

The traffic accident rate in the middle section of the extra long tunnel was lower than the average tunnel accident rate. This was mainly because the driver became more adapted to the internal environment of the tunnel after passing through the tunnel entrance section [7]. Additionally, the driver was more cautious since the tunnel exit was still far away.

\subsection{Extra Long Tunnel Traffic Safety Affected Zone Classification}

Based on the driving behavior characteristics of the extra long tunnel and the longitudinal distribution characteristics of traffic accidents, with reference to the requirements for the lighting setting requirements of the one-way traffic tunnel 
in the "Guidelines for Design of Lighting of Highway Tunnels" (JTGT D70/2-01-2014), the extra long tunnel was divided into five tunnel sections, including the tunnel entrance affected zone, the tunnel entrance section, the tunnel middle section, the tunnel exit section, and the tunnel exit affected zone.

The tunnel entrance affected zone: $200 \mathrm{~m}$ prior to extra long tunnel entrance.

The tunnel entrance section: extra long tunnel entrance to the tunnel section $300 \mathrm{~m}$.

The tunnel middle section: the remaining length of the extra long tunnel with the removal of the tunnel entrance section and the tunnel exit section.

The tunnel exit section: 100 meters long before the tunnel exit to the extra long tunnel exit.

The tunnel Exit affected zone: $300 \mathrm{~m}$ outside the extra long tunnel exit.

\section{Analysis of the Relationship between the Number of Accidents and the Characteristics of the Traffic Flow}

\subsection{Relationship between Accidents and Traffic Flow}

The traffic flow takes 100 vehicles per hour as the step size; the proportion of large vehicles and the lane occupancy rate are both taken as the step size. The statistical analysis of the number of accidents in extra long tunnel is as follows.

As shown in Figure 5, the traffic flow is in the range of 1000 vehicles/h and 1500 vehicles/h, the frequency of traffic accidents is high, and the number of traffic accidents is higher than 10 times; when the traffic flow is in the range of 1500 vehicles/h and 3600 vehicles/h, the number of traffic accidents is almost stable, and the number of traffic accidents was stable and less than 3 times; when the traffic flow is higher than 3600 vehicles/h, the number of traffic accidents increased volatilely.

As shown in Figure 6, the proportion of large vehicles has always been one of the important factors affecting the safety of traffic operations. When the proportion of large vehicles is $0.15-0.20$, there is a clear peak in the number of traffic accidents. If the proportion is less than 0.15 , with the increase of the proportion of large vehicles, the number of traffic accidents shows a volatile growth trend; in the range of greater than 0.20 , the growth trend of traffic accidents has slowed down.

As shown in Figure 7, the lane occupancy is an important indicator of the degree of road congestion. A higher occupancy rate indicates that there are more vehicles on the lane and greater mutual interference between vehicles. When the occupation rate is in the range of $0.03-0.05$, the number of traffic accidents has a significant peak. When the occupation rate is less than 0.03 , the number of traffic accidents has increased significantly with the increase in the occupancy rate; if the occupation rate is greater than 0.05 , the number of traffic accidents has basically stabilized, and the number of traffic accidents is less than 5 . When the occupancy rate increased to 0.47 during the observation period, the number of traffic accidents increased significantly. 


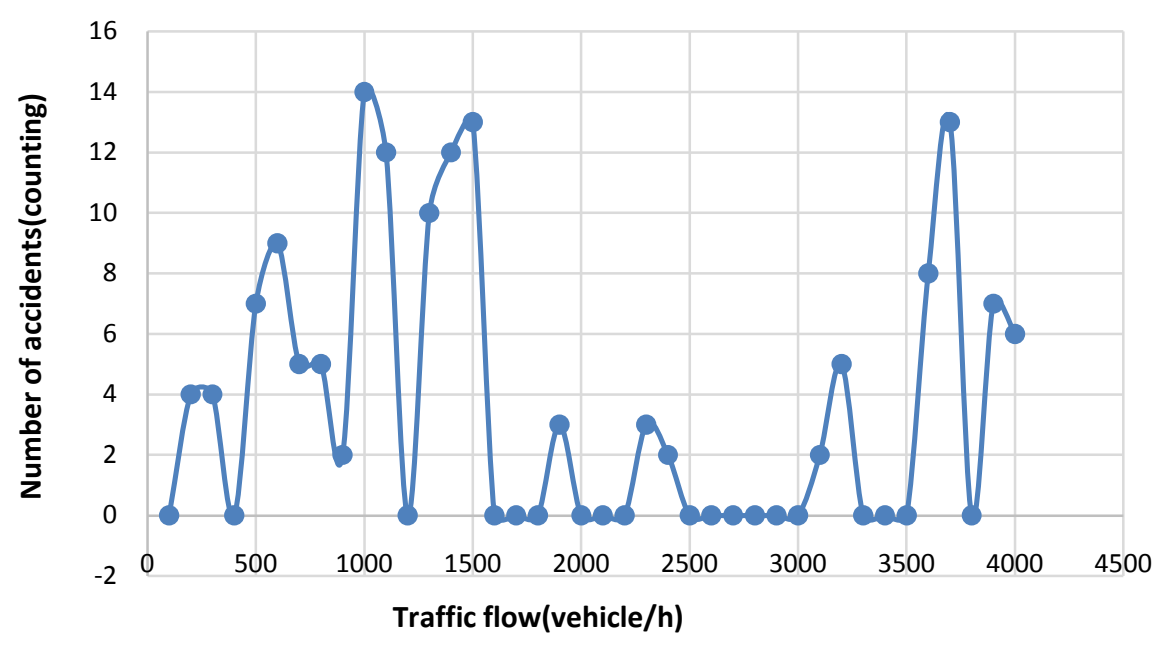

Figure 5. Relationship between the number of accidents and traffic flow.

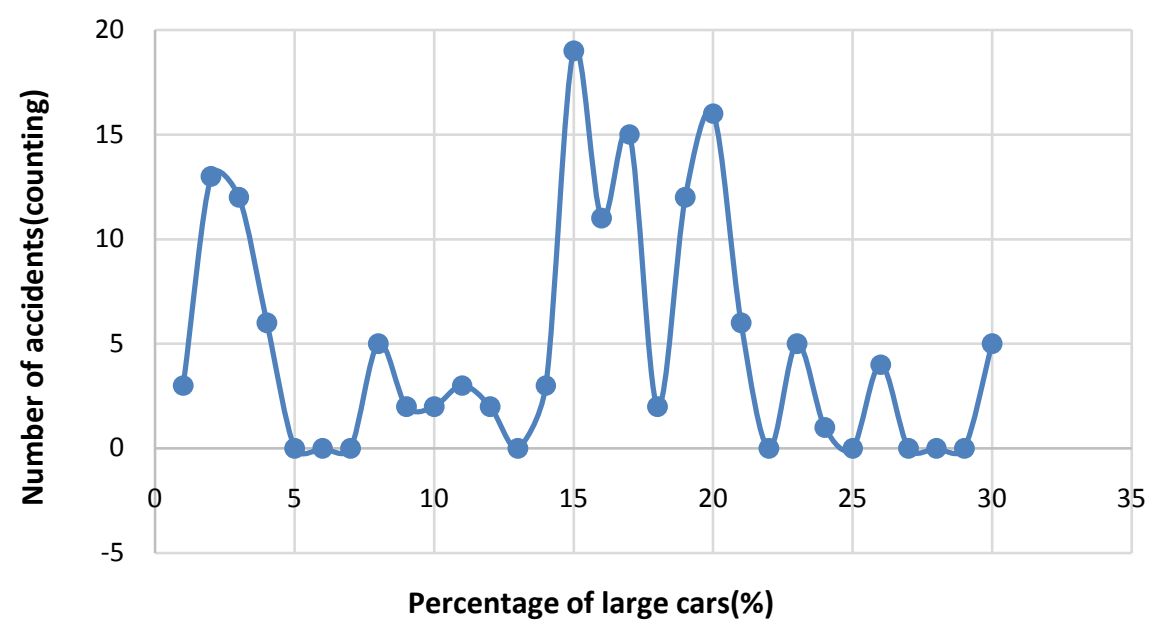

Figure 6. Relationship between the number of accidents and the proportion of large vehicles.

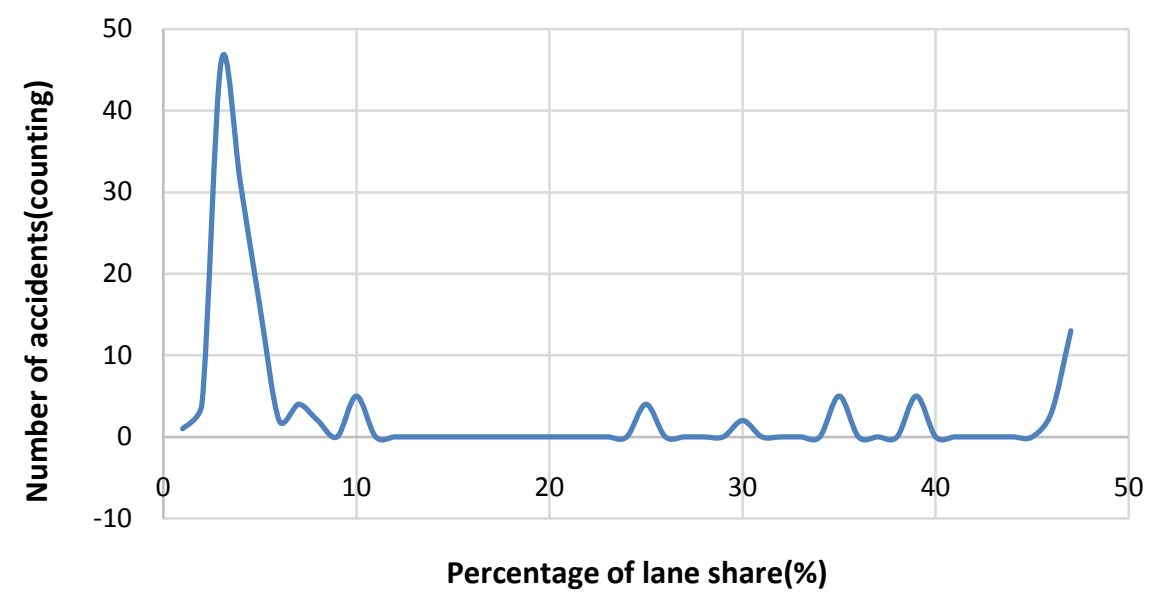

Figure 7. Relationship between the number of accidents and lane share.

\subsection{Relationship between Accidents and Average Speed}

The average speed of the traffic flow within the hour of the accident is selected 
as the speed beam index of the traffic flow before and after the accident, and the $5 \mathrm{~km} / \mathrm{h}$ is used as the statistical step to analyze the number of accidents in each speed section. It can be seen from Figure 8 that the traffic flow speed is in the range of $20 \mathrm{~km} / \mathrm{h}-30 \mathrm{~km} / \mathrm{h}$, there is a clear peak period of traffic accidents; when the traffic flow speed in the range of $30 \mathrm{~km} / \mathrm{h}-75 \mathrm{~km} / \mathrm{h}$, the number of accidents is in a relatively stable period, and the number of traffic accidents is less than 3; When the traffic flow speed is greater than $75 \mathrm{~km} / \mathrm{h}$, the number of traffic accidents increases rapidly. When the traffic flow speed reaches $85 \mathrm{~km} / \mathrm{h}$ during the observation period, the number of traffic accidents reaches up to 80 .

\section{Analysis of Relationship between Traffic Accidents and Longitudinal Position}

\subsection{Time Distribution of Accidents in Different Locations}

It can be seen from Figure 9 that the traffic accident rate was low during the period of $22 \mathrm{pm}$ to $6 \mathrm{am}$. The traffic accidents that occurred within 8 hours accounted for $4.76 \%$ of the total number of 24 -hour accidents. The traffic flow during this period was relatively small, accounting $7.5 \%$ for 24 hours of traffic flow. Traffic accidents occurred frequently at 7 - 18 hours, accounting for $89.8 \%$ of the total number of traffic accidents. Among them, traffic accidents were intensive between $11 \mathrm{am}-12 \mathrm{pm}$ and $14 \mathrm{pm}-15 \mathrm{pm}$, and the 2-hour accident rate accounted for $24.49 \%$ of the total number of 24 -hour traffic accidents [8].

\subsection{Patterns Distribution of Accident in Different Locations}

As shown in Figure 10 that the accidents in extra long tunnel accounted for $70.4 \%$ of the total number of tunnel traffic accidents. Among them, the rear-end accidents occurred frequently, accounting for $29.4 \%$ of the total number of tunnel traffic accidents. The accidents caused by the same direction scraping and other angle collisions accounted $12.6 \%$ and $28.5 \%$ for tunnel traffic accidents

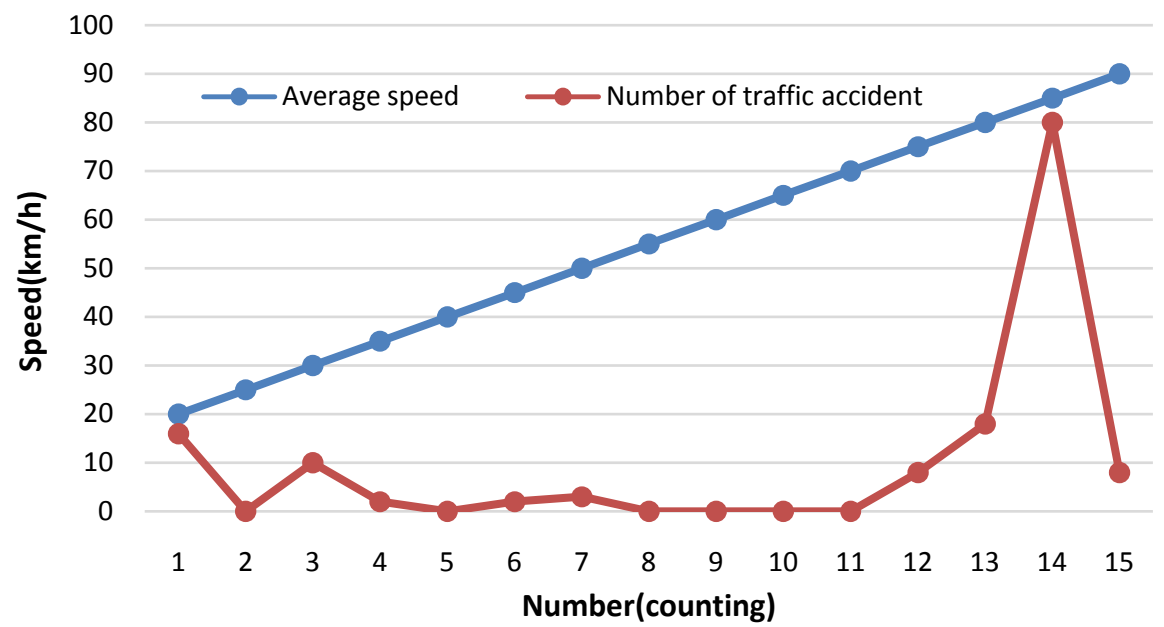

Figure 8. Relationship between the number of accidents and average speed. 


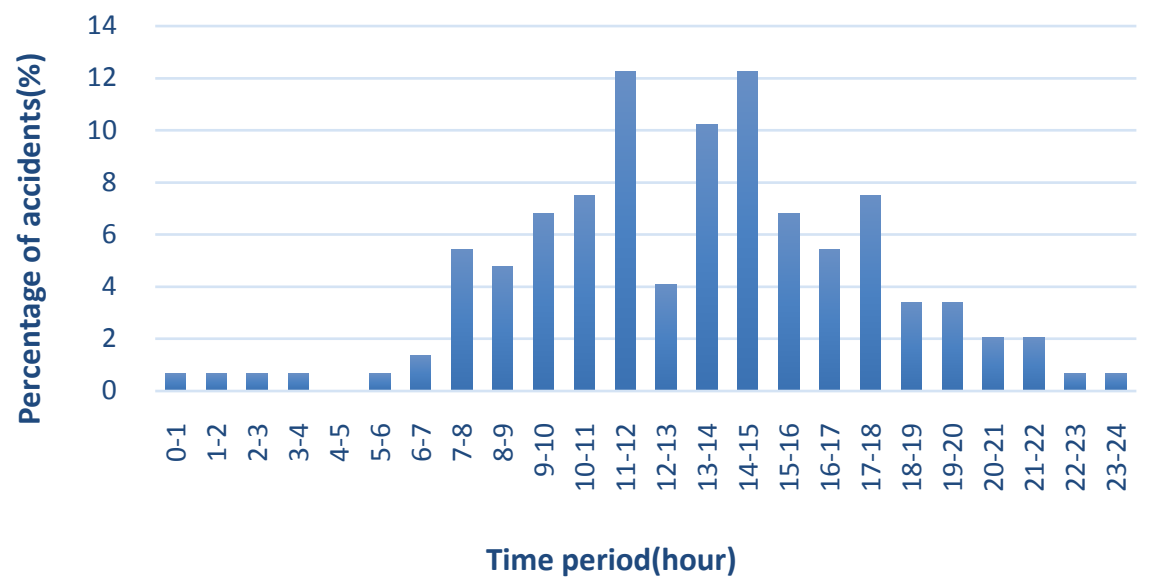

Figure 9. Distribution 24 hours of traffic accident in extra long tunnel.

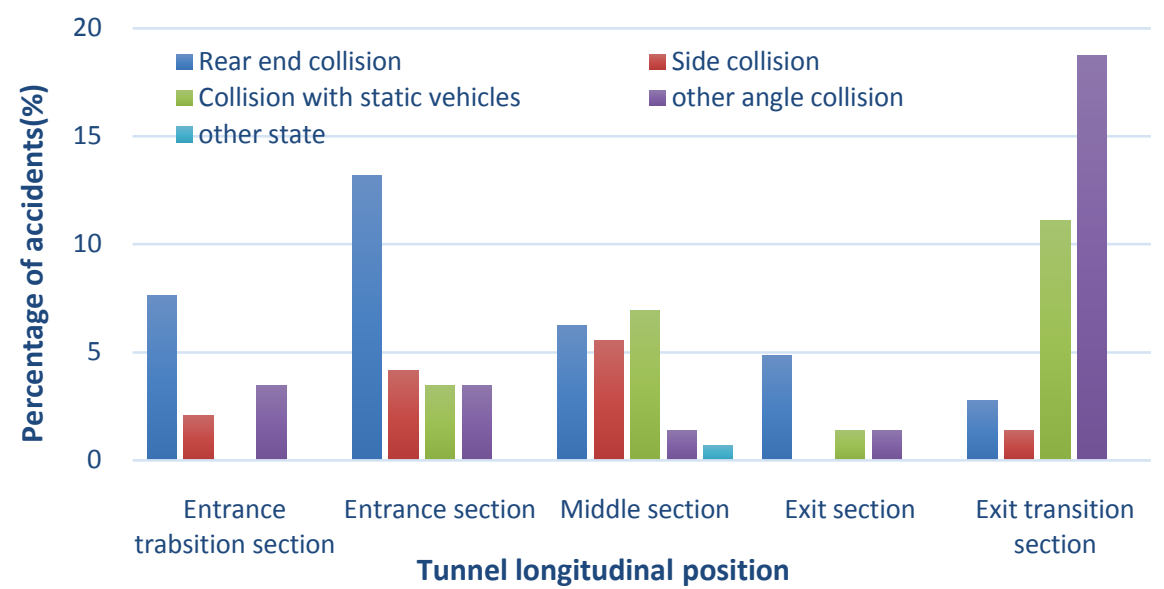

Figure 10. Distribution of traffic accident state in extra long tunnel.

respectively. Single vehicle accidents accounted for $29.4 \%$ of the total number of tunnel traffic accidents, where collision with static vehicles was the main reason. Second, other inter-vehicle accidents accounted for $1.6 \%$ of the total number of tunnel traffic accidents.

In the tunnel, the inter-vehicle accidents and rear-end collision accidents at the tunnel entrance section accounted for $77.5 \%$ and $50.7 \%$ of the total number of tunnel entrance accidents respectively; the inter-vehicle accidents and rear-end collision accidents at the tunnel exit section accounted for the total number of accidents at the tunnel exit section for $62.3 \%$ and $34.9 \%$ respectively. Outside the tunnel, the traffic accident pattern within $2 \mathrm{~km}$ of the tunnel exit affected zone was mainly due to other angle collisions, accounting for $49.3 \%$ of the total number of accidents in the tunnel exit affected area.

\subsection{Cause Distribution of Accidents in Different Locations}

As shown in Figure 11, the proportion of traffic accidents caused by illegal lane change was relatively high, accounting for $15.79 \%$ of the total traffic accidents in the tunnel entrance affected zone; compared to other sections, the proportion of 


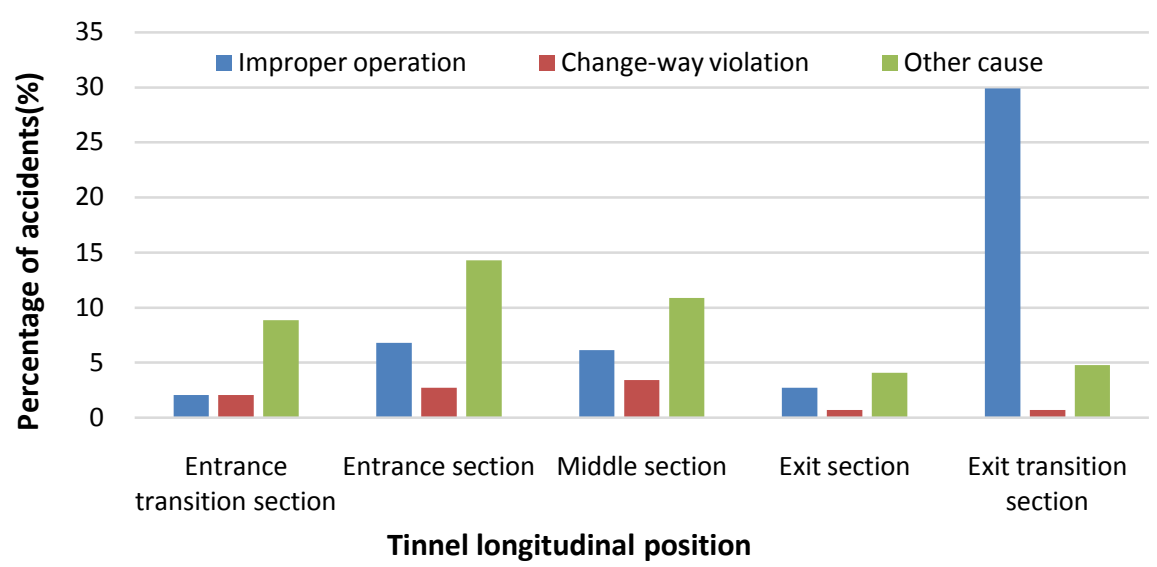

Figure 11. Distribution of traffic accidents in different sections of extra long tunnel.

traffic accidents caused by improper operation and other safety incidents in tunnel entrance section was higher, that accounts for $6.81 \%$ and $14.29 \%$ of the total number of tunnel traffic accidents respectively; in the middle of the tunnel, the proportion of traffic accidents caused by illegal lane change was relatively high as well, accounting for $3.40 \%$ of the total number of tunnel traffic accidents; The proportion of traffic accidents was low, accounting for $0.68 \%$ of the total number of tunnel traffic accidents. In the tunnel exit affected zone, the proportion of traffic accidents caused by improper operation was high, accounting for $29.93 \%$ of the total number of tunnel traffic accidents.

\section{Conclusions}

Based on the systematic investigation of the driving environment of the extra long tunnel, this paper expounds the characteristics of the running speed and the characteristics of traffic accidents in different sections of the extra long tunnel, analyzes the potential influencing factors and causes, and draws the following conclusions:

1) Based on the longitudinal distribution characteristics of operating speed and traffic accidents, the extra long tunnel can be divided into five traffic safety affected zones: tunnel entrance affected zone, tunnel entrance section, tunnel middle section, tunnel exit section and tunnel exit affected zone.

2) The number of accidents tends to be stable when the traffic volume of extra long tunnel is between 1500 vehicles/h and 3600 vehicles/h, and the number of traffic accidents is higher when the proportion of large vehicles is $15 \%-20 \%$. When the lane occupation rate is higher than $5 \%$, the number of traffic accidents is less than 5 times.

3) The longitudinal distribution of traffic accidents in extra long tunnel is quite different. The incidence of traffic accidents in the entrance and exit affected zones is relatively high, and the incidence of traffic accidents in the middle section is relatively low.

4) The type of traffic accidents in extra long tunnel is mainly due to rear-end collisions and collision with static vehicles. Small passenger cars and heavy 
goods vehicles are the main types of accidents, and most of them are bicycle accidents.

5) The proportion of traffic accidents caused by improper operation, illegal lane change and obstruction of safe driving behavior in extra long tunnel traffic accidents is high and those factors are also the major reasons for in tunnel accidents in extra long tunnel environment.

\section{Acknowledgements}

This research was jointly supported by the Chinese National Natural Science Foundation (51708421), and scientific research project of STCSM (Science and Technology Commission of Shanghai Municipality) 17D1203302.

\section{Conflicts of Interest}

The authors declare no conflicts of interest regarding the publication of this paper.

\section{References}

[1] Chen, L. (2014) Analysis of Traffic Accidents in Expressway Tunnels. Journal of Road Traffic \& Transportation Technology, 8, 259-261.

[2] Ministry of Transport of the People's Republic of China (2016) Statistical Bulletin on the Development of Transport Industry in 2016.

[3] Zhou, J.J., Wang, J. and Lu, L.J. (2019) Research on Statistical Analysis of Traffic Accident Characteristics in the Urban River-Crossing Tunnel. Urban Passenger Transportation, 2019, 75-79.

[4] Yeung, J.S. and Wong, Y.D. (2013) Road Traffic Accidents in Singapore Expressway Tunnels. Tunnelling and Underground Space Technology, 38, 534-541. https://doi.org/10.1016/j.tust.2013.09.002

[5] Tanishita, M. and van Wee, B. (2017) Impact of Vehicle Speeds and Changes in Mean Speeds on per Vehicle-Kilometer Traffic Accident Rates in Japan. IATSS Research, 41, 107-112. https://doi.org/10.1016/j.iatssr.2016.09.003

[6] Zhang, S.-R., Ma, Z.-L. and Shi, Q. (2007) Distribution Characteristics of Traffic Accidents in Expressway Tunnel Groups and Countermeasures for Prevention. Journal of Chang' an University (Natural Science Edition), 27, 63-66.

[7] Zhang, Y.-C., He, C., Wu, D.-X. and Zeng, Y.-H. (2009) Traffic Accidents in Expressway Tunnels and Their Prevention Measures. Journal of SouthWest Jiaotong University, 10, 776-781.

[8] Wang, B.X. (2012) The Traffic Accident Characteristics Analysis of Highway Tunnel. Traffic \& Transportation, 7, 164-167. 